\title{
Binormal Motion of Curves with Constant Torsion in 3-Spaces
}

\author{
Josu Arroyo, Óscar J. Garay, and Álvaro Pámpano \\ Department of Mathematics, Faculty of Science and Technology, University of the Basque Country, Apartado 644, 48014 Bilbao, Spain \\ Correspondence should be addressed to Óscar J. Garay; oscarj.garay@ehu.eus
}

Received 3 May 2017; Accepted 30 May 2017; Published 29 June 2017

Academic Editor: Andrei D. Mironov

Copyright (C) 2017 Josu Arroyo et al. This is an open access article distributed under the Creative Commons Attribution License, which permits unrestricted use, distribution, and reproduction in any medium, provided the original work is properly cited.

\begin{abstract}
We study curve motion by the binormal flow with curvature and torsion depending velocity and sweeping out immersed surfaces. Using the Gauss-Codazzi equations, we obtain filaments evolving with constant torsion which arise from extremal curves of curvature energy functionals. They are "soliton" solutions in the sense that they evolve without changing shape.
\end{abstract}

\section{Introduction}

A large class of physical systems are modelled in terms of motion of curves and surfaces in Euclidean space $\mathbb{R}^{3}$. A remarkable example is the so-called localized induction equation (LIE)

$$
x_{t}=x_{s} \times x_{s s}
$$

which is a soliton equation used to model the dynamics of a thin vortex filament in an incompressible, inviscid, homogeneous, 3-dimensional fluid [1-3]. Quite often, by resorting to the underlying geometry one can gain considerable insight into the dynamics of physical systems $[3,4]$. Here, we use a geometrical approach to investigate an extension of (1) obtained by considering a smooth map $x: U \subset \mathbb{R}^{2} \rightarrow M_{r}^{3}(\rho)$, $x(s, t)$, verifying

$$
x_{t}=f\left(\left|\widetilde{\nabla}_{x_{s}} x_{s}\right|, \frac{\operatorname{det}\left(x_{s}, \widetilde{\nabla}_{x_{s}} x_{s}, \widetilde{\nabla}_{x_{s}}^{2} x_{s}\right)}{\left|\widetilde{\nabla}_{x_{s}} x_{s}\right|^{2}}\right) x_{s} \times \widetilde{\nabla}_{x_{s}} x_{s} \text {, }
$$

where $f$ is a suitable smooth function, $\widetilde{\nabla}$ denotes the LeviCivita connection on $M_{r}^{3}(\rho), r \in\{0,1\}$, and $M_{r}^{3}(\rho)$ is a Riemannian $(r=0)$ or Lorentzian $(r=1) 3$-space form with constant curvature $\rho$; that is, $M_{r}^{3}(\rho)$ is one of the following: $\mathbb{R}^{3}$, the sphere $\mathbb{S}^{3}$, the hyperbolic space $\mathbb{H}^{3}$, the Minkowski space $\mathbb{R}_{1}^{3}$, the de Sitter space $\mathbb{S}_{1}^{3}$, or the anti de Sitter space $\mathbb{M}_{1}^{3}$.

Under mild conditions we will see that a curve motion following (2) describes a curve $\gamma$ evolving under the binormal flow, with velocity depending on curvature and torsion (19), and determines an immersed surface, $S_{\gamma}$, in $M_{r}^{3}(\rho)$. Then, fundamental results of the theory of submanifolds can be applied and it will be seen that solving geometrically (2) amounts to solving the Gauss-Codazzi equations (40) and (41), since that would give us the curvature and torsion of a geodesic foliation of $S_{\gamma}$. Alternatively, one can determine the evolution by finding a single solution, working as initial condition $x(s, 0)=\gamma(s)$, and then giving a geometrical description of the binormal flow.

If $M_{r}^{3}(\rho)=\mathbb{R}^{3}$ and $f \equiv 1,(2)$ reduces to LIE (1) and it can be seen that Gauss-Codazzi equations boil down to $\mathrm{Da}$ Rios equations found in 1906 [2]. In Lorentzian backgrounds (1) has been studied in $[1,5]$, while long time existence of closed solutions in Riemannian ambient spaces are analyzed in [6]. If $f \equiv f\left(\left|\widetilde{\nabla}_{x_{s}} x_{s}\right|\right)$, travelling wave solutions of the Gauss-Codazzi equations have been investigated in [7]. In the second part of this work, we focus on curves evolving by (2) with constant torsion and use the Gauss-Codazzi equations to construct solutions by means of extremal curves for curvature dependent energies and associated 1-parameter groups of isometries.

\section{Preliminaries}

Consider the Euclidean semispace $\mathbb{E}_{v}^{m}$, that is, $\mathbb{R}^{m}$ endowed with the canonical metric of index $\nu$, denoted by $\langle\cdot, \cdot\rangle$, and the Levi-Civita connection, denoted by $\bar{\nabla}$. Then, the semiRiemannian 3-space forms $M_{r}^{3}(\rho)$ (the Riemannian case, 
$r=0$ will be simply denoted by $\left.M^{3}(\rho)\right)$ can be isometrically immersed in $\mathbb{E}_{v}^{4}$, the 4-dimensional Euclidean semispace, in a standard way [8]. The flat case, $M_{r}^{3}(\rho)=\mathbb{E}_{r}^{3}, \rho=0, r=0,1$, corresponds to either $\mathbb{R}^{3}$ or the Minkowski space $\mathbb{R}_{1}^{3} \equiv \mathbb{L}^{3}$. They can be isometrically immersed in $\mathbb{L}^{4}=\mathbb{R}_{1}^{4}$ endorsed with the metric

$$
g=d x_{1}^{2}+d x_{2}^{2}+d x_{3}^{2}-d x_{4}^{2}
$$

in an obvious manner:

$$
\begin{aligned}
& \mathbb{R}^{3}=\left\{\left(x_{1}, x_{2}, x_{3}, x_{4}\right) \in \mathbb{L}^{4} \mid x_{4}=0\right\}, \\
& \mathbb{L}^{3}=\left\{\left(x_{1}, x_{2}, x_{3}, x_{4}\right) \in \mathbb{L}^{4} \mid x_{1}=0\right\} .
\end{aligned}
$$

When $\rho>0, M_{r}^{3}(\rho)$ correspond to the 3-sphere, $\mathbb{S}^{3}(\rho)(r=$ $0)$, and the de Sitter 3 -space, $\mathbb{S}_{1}^{3}(\rho)(r=1)$, defined by

$$
\mathbb{S}_{r}^{3}(\rho)=\left\{\mathbf{x} \in \mathbb{E}_{r}^{4} \mid\langle\mathbf{x}, \mathbf{x}\rangle=\frac{1}{\rho}\right\},
$$

where $\mathbf{x}=\left(x_{1}, x_{2}, x_{3}, x_{4}\right)$. Finally, for $\rho<0$ we obtain the hyperbolic 3-space, $\mathbb{M}^{3}(r=0)$, and the anti de Sitter 3-space, $\mathbb{H}_{1}^{3}(r=1)$

$$
\mathbb{H}_{r}^{3}(\rho)=\left\{\mathbf{x} \in \mathbb{E}_{r+1}^{4} \mid\langle\mathbf{x}, \mathbf{x}\rangle=\frac{1}{\rho}\right\} .
$$

The standard isometric immersions of $M_{r}^{3}(\rho)$ into $\mathbb{E}_{v}^{4}$ ([8] p. 20) will be all denoted by $i$ and the induced metrics also by $\langle\cdot, \cdot\rangle$, while the Levi-Civita connections on $\mathbb{E}_{\nu}^{4}$ and $M_{r}^{3}(\rho)$ are denoted by $\bar{\nabla}$ and $\widetilde{\nabla}$, respectively. As usual, the cross product of two vector fields $X, Y$ in $M_{r}^{3}(\rho)$, denoted by $X \times Y$, is defined so that $\langle X \times Y, Z\rangle=\operatorname{det}(X, Y, Z)$ for any other vector field $Z$ of $M_{r}^{3}(\rho)$, where $\operatorname{det}(X, Y, Z)$ stands for the determinant.

Now, for a given isometric immersion of a surface, $x$ : $N_{v}^{2} \rightarrow M_{r}^{3}(\rho), v \in\{0,1\}$, we denote by $\nabla$ the Levi-Civita connection of the immersion $\left(N_{v}^{2}, x\right)$. As it is also customary, for a surface $N_{v}^{2}$ in any 3-dimensional space form $M_{r}^{3}(\rho)$, we require the first fundamental form to be nondegenerate. Take $X, Y, Z, W$ tangent vector fields to $N_{v}^{2}$ and choose $\xi$ a normal vector field to $N_{v}^{2}$ in $M_{r}^{3}(\rho)$. Then the formulas of Gauss and Weingarten are, respectively, as follows [8]:

$$
\begin{aligned}
\bar{\nabla}_{X} Y & =\widetilde{\nabla}_{X} Y-\rho\langle X, Y\rangle x \\
& =\nabla_{X} Y+h(X, Y)-\rho\langle X, Y\rangle x, \\
\widetilde{\nabla}_{X} \xi & =-A_{\xi} X+D_{X}^{\perp} \xi,
\end{aligned}
$$

where $x=i \circ x$ is the position vector, $h$ denotes the second fundamental form of $N_{v}^{2}$ in $M_{r}^{3}(\rho), A_{\xi}$ stands for the Weingarten map, and $D^{\perp}$ denotes the connection on the normal bundle of $N_{v}^{2}$. By using (7) and (8) and denoting by $R$ and $\widetilde{R}$ the Riemann curvature tensors associated with $\nabla$ and $\widetilde{\nabla}$, respectively, the following relation holds:

$$
\widetilde{R}(X, Y) Z=\rho(\langle Y, Z\rangle X-\langle X, Z\rangle Y),
$$

while the equations of Gauss and Codazzi are given, respectively, by [8]

$$
\begin{aligned}
\langle\widetilde{R}(X, Y) Z, W\rangle= & \langle R(X, Y) Z, W\rangle \\
& -\langle h(\mathrm{X}, W), h(Y, Z)\rangle \\
& +\langle h(X, Z), h(Y, W)\rangle, \\
(\nabla h)(X, Y, Z)= & (\nabla h)(Y, X, Z),
\end{aligned}
$$

where $\nabla h$ is defined by

$$
\begin{aligned}
(\nabla h)(X, Y, Z)= & D_{X}^{\perp} h(Y, Z)-h\left(\nabla_{X} Y, Z\right) \\
& -h\left(Y, \nabla_{X} Z\right)
\end{aligned}
$$

Now choosing an adapted local orthonormal frame $\left\{e_{1}, e_{2}, e_{3}\right\}$ in $M_{r}^{3}(\rho)$ such that the vectors $e_{1}, e_{2}$ are tangent to $N_{v}^{2}$ and $e_{3}$ is normal to $N_{v}^{2}$ in $M_{r}^{3}(\rho)$, and denoting by $\left\{\omega^{1}, \omega^{2}, \omega^{3}\right\}$ the dual frame of $\left\{e_{1}, e_{2}, e_{3}\right\}$, the Cartan connection forms are defined by

$$
\widetilde{\nabla}_{X} e_{i}=\sum_{j} \widetilde{\varepsilon}_{j} \omega_{i}^{j}(X) e_{j}
$$

for $i, j \in\{1,2,3\}$, where $\widetilde{\varepsilon}_{j}=\left\langle e_{j}, e_{j}\right\rangle$ is the causal character of $e_{j}$. Then, $\omega_{i}^{j}=-\omega_{j}^{i}$ and

$$
\begin{aligned}
h\left(e_{i}, e_{j}\right) & =\widetilde{\varepsilon}_{3} h_{i j} e_{3}, \\
h_{i j} & =-\left\langle\widetilde{\nabla}_{e_{i}} e_{3}, e_{j}\right\rangle=\omega_{j}^{3}\left(e_{i}\right),
\end{aligned}
$$

$i, j \in\{1,2\}$ [8]. We will often resort to the standard abuse of notation and identification tricks in submanifold theory.

\section{Binormal Evolution Surfaces}

The covariant derivative of a vector field $X$ along a curve $\gamma$ will be denoted by $(D X / d s)(s):=\widetilde{\nabla}_{T} X(s)$. Let $\gamma(s)$ be a unit speed nongeodesic curve immersed in $M_{r}^{3}(\rho)$ with nonnull velocity $(D \gamma / d s)(s)=T(s), \forall s$; therefore, it is assumed to be either spacelike or timelike. If it also has nonnull acceleration $(D T / d s)(s)$, then $\gamma(s)$ is a Frenet curve of rank 2 or 3 and the standard Frenet frame along $\gamma(s)$ is given by $\{T, N, B\}(s)$, where $B$ is chosen so that $\operatorname{det}(T, N, B)=1$. Then the Frenet equations define the curvature, $\kappa(s)$, and torsion, $\tau(s)$, along $\gamma(s)$

$$
\begin{aligned}
& \frac{D T}{d s}(s)=\varepsilon_{2} \kappa(s) N(s), \\
& \frac{D N}{d s}(s)=-\varepsilon_{1} \kappa(s) T(s)+\varepsilon_{3} \tau(s) B(s), \\
& \frac{D B}{d s}(s)=-\varepsilon_{2} \tau(s) N(s),
\end{aligned}
$$


where $\varepsilon_{i}, 1 \leq i \leq 3$, is the causal character of $T, N$, and $B$, respectively. Notice that the following relations hold:

$$
\begin{gathered}
T=\varepsilon_{1} N \times B, \\
N=\varepsilon_{2} B \times T, \\
B=\varepsilon_{3} T \times N .
\end{gathered}
$$

Curves for which both curvature and torsion are constant are called Frenet helices. In a semi-Riemannian space form any local geometrical scalar defined along Frenet curves can always be expressed as a function of their curvatures and derivatives.

Given a smooth map $x: U \subset \mathbb{R}^{2} \rightarrow M_{r}^{3}(\rho), x(s, t)$, satisfying (2), we usually identify $x$ and $i \circ x$. Assume that the initial condition $\gamma(s):=x(s, 0)$ is a unit speed Frenet curve of rank 2 or 3 ; then $\gamma^{t}(s):=x(s, t)$, which will be called the filament at time $t$, is also unit speed parametrized $\forall t$. In fact, we have $(\partial / \partial t)\left\langle x_{s}(s, t), x_{s}(s, t)\right\rangle=2\left\langle\widetilde{\nabla}_{x_{s}} x_{t}, x_{s}\right\rangle=$ 0 , where the last equality is obtained from (2). So, since $\left\langle x_{s}(s, 0), x_{s}(s, 0)\right\rangle=\langle d \gamma / d s, d \gamma / d s\rangle=\langle T, T\rangle=\varepsilon_{1}$, then so is $\forall t$; that is, (2) is a length-preserving evolution. Assuming also that $\widetilde{\nabla}_{x_{s}} x_{s}(s, t)$ is nonnull everywhere, the associated Frenet frame will be defined for all $\gamma^{t}$ and combining (2) and (15) we obtain

$$
\begin{aligned}
x_{t} & =f(\kappa, \tau) x_{s} \times \widetilde{\nabla}_{x_{s}} x_{s}=f(\kappa, \tau) T \times \frac{D T}{d s} \\
& =\varepsilon_{2} \kappa f(\kappa, \tau) T \times N=\varepsilon_{2} \varepsilon_{3} \kappa f(\kappa, \tau) B=\mathscr{F}(\kappa, \tau) B .
\end{aligned}
$$

This means that $\gamma(s)$ evolves by the binormal flow with velocity $\mathscr{F}(\kappa, \tau)$. We are going to suppose also that $f$ is never zero so that $(U, x)$ defines an immersed surface in $M_{r}^{3}(\rho)$ swept out by $\gamma(s)$. It will be denoted by $S_{\gamma}$ and called a binormal evolution surface (BES) with initial condition $\gamma$ and velocity $\mathscr{F}$. The curves $\gamma^{s}(t):=x(s, t)$, perpendicular to the filaments, are called fibers of $S_{\gamma}$. The time variation of the Frenet frames is described in the following proposition (cf. (3.15) of [3] for surfaces in $\mathbb{R}^{3}$ ).

Proposition 1. Let $(U, x)$ be a BES of $M_{r}^{3}(\rho)$ with velocity $\mathscr{F}$ (19). Then

$$
\begin{aligned}
& \tilde{\nabla}_{\partial / \partial s}\left(\begin{array}{c}
T \\
N \\
B
\end{array}\right)(s, t)=\left(\begin{array}{ccc}
0 & \kappa & 0 \\
-\kappa & 0 & \tau \\
0 & -\tau & 0
\end{array}\right)\left(\begin{array}{l}
\varepsilon_{1} T \\
\varepsilon_{2} N \\
\varepsilon_{3} B
\end{array}\right)(s, t), \\
& \tilde{\nabla}_{\partial / \partial t}\left(\begin{array}{c}
T \\
N \\
B
\end{array}\right)(s, t) \\
& =\left(\begin{array}{ccc}
0 & -\tau \mathscr{F} & \varepsilon_{3} \mathscr{F}_{s} \\
\tau \mathscr{F} & 0 & \varepsilon_{2} h_{22} \mathscr{F} \\
-\varepsilon_{3} \mathscr{F}_{s} & -\varepsilon_{2} h_{22} \mathscr{F} & 0
\end{array}\right)\left(\begin{array}{l}
\varepsilon_{1} T \\
\varepsilon_{2} N \\
\varepsilon_{3} B
\end{array}\right)(s, t),
\end{aligned}
$$

where $h_{22}=(1 / \kappa)\left\{\varepsilon_{3}\left(\mathscr{F}_{s s} / \mathscr{F}\right)-\varepsilon_{2} \tau^{2}+\varepsilon_{1} \varepsilon_{3} \rho\right\}$ and $\kappa(s, t)$ and $\tau(s, t)$ denote the curvature and torsion of the curves $\gamma^{t}(s)$.
Proof. Under our assumptions, all $\gamma^{t}$ are unit speed parametrized and they all have well defined Frenet frame satisfying (15)-(17), so (20) is clear. As for the second part, since $\gamma^{t}(s)=x(s, t)$ is not a geodesic in $M_{r}^{3}(\rho)$ and $\widetilde{\nabla}_{x_{s}} x_{s}$ is not null, then, for sufficiently small $s$, the unit Frenet normal to $\gamma^{t}(s), N(s, t)$, is parallel to a (local) unit normal to $S_{\gamma}$. This means that $\gamma^{t}(s)$ are geodesics in $S_{\gamma}$ for any $t$ and the parametrization

$$
x(s, t)=\gamma^{t}(s)
$$

determines a geodesic coordinate system with respect to which the metric $\langle,\rangle \equiv g$ can be written as

$$
g=\varepsilon_{1} d s^{2}+\varepsilon_{3} \mathscr{F}^{2} d t^{2}
$$

Now, the Gauss and Weingarten formulas (7) and (8), in combination with the Gauss and Codazzi equations (10) and (11), will give us all the relevant geometric information about the immersion $(U, x)$. This requires bringing in some computational stuff and very long calculations whose details are omitted here. Thus, the Christoffel symbols of the LeviCivita connection of (23) with respect to the parametrization (22) (see, e.g., [8], Proposition 1.1) can be computed from the metric coefficients $g_{i j}$. In our case, we have

$$
\begin{aligned}
\Gamma_{11}^{1} & =\Gamma_{11}^{2}=\Gamma_{12}^{1}=0, \\
\Gamma_{12}^{2} & =\frac{\mathscr{F}_{s}}{\mathscr{F}}, \\
\Gamma_{22}^{1} & =-\varepsilon_{1} \varepsilon_{3} \mathscr{F}_{\mathscr{F}_{s}}, \\
\Gamma_{22}^{2} & =\frac{\mathscr{F}_{t}}{\mathscr{F}},
\end{aligned}
$$

where subscripts $s$ and $t$ mean partial derivative with respect to $s$ and $t$, respectively. This makes it possible to know the expression for the Levi-Civita connection of $S_{\gamma}([8], \$ 1.4)$, denoted here by $\nabla$

$$
\begin{aligned}
& \nabla_{\partial / \partial s} \frac{\partial}{\partial s}=0, \\
& \nabla_{\partial / \partial s} \frac{\partial}{\partial t}=\frac{\mathscr{F}_{s}}{\mathscr{F}} \frac{\partial}{\partial t}, \\
& \nabla_{\partial / \partial t} \frac{\partial}{\partial t}=-\varepsilon_{1} \varepsilon_{3} \mathscr{F} \mathscr{F}_{s} \frac{\partial}{\partial s}+\frac{\mathscr{F}_{t}}{\mathscr{F}} \frac{\partial}{\partial t} .
\end{aligned}
$$

As before, $\{T(s, t), N(s, t), B(s, t)\}$ represent the Frenet frames along $\gamma^{t}(s)$, and we choose the following local adapted frame on $S_{\gamma}$ :

$$
\begin{aligned}
& e_{1}=x_{s}=T, \\
& e_{2}=\frac{x_{t}}{\mathscr{F}}=B, \\
& e_{3}=\xi=-\varepsilon_{2} N,
\end{aligned}
$$


where $\xi$ is the unit normal to $S_{\gamma}$ (locally defined). Then, combining (7), (8), (13), (14), and (15)-(17), one gets

$$
\begin{aligned}
& \omega_{1}^{2}\left(e_{1}\right)=0, \\
& \omega_{1}^{2}\left(e_{2}\right)=\varepsilon_{3} \frac{\mathscr{F}_{s}}{\mathscr{F}}, \\
& \omega_{1}^{3}\left(e_{1}\right)=h_{11}=-\varepsilon_{2} \kappa, \\
& \omega_{1}^{3}\left(e_{2}\right)=\omega_{2}^{3}\left(e_{1}\right)=h_{12}=\varepsilon_{2} \tau, \\
& \omega_{2}^{3}\left(e_{2}\right):=h_{22},
\end{aligned}
$$

where $\kappa(s, t)$ and $\tau(s, t)$ denote the curvature and torsion of the curves $\gamma^{t}(s)$.

The second fundamental form can be considered as a quadratic form given by $h(X):=\left\langle A_{\xi} X, X\right\rangle$; therefore, we obtain from (27) that

$$
h=-\varepsilon_{2} \kappa d s^{2}+2 \varepsilon_{2} \tau \mathscr{F} d s d t+\mathscr{F}^{2} h_{22} d t^{2},
$$

with respect to the parametrization (22), where $h_{22}$ is the coefficient of the second fundamental form ([8], \$2.3) of $S_{\gamma}$ in $M_{r}^{3}(\rho)$ given by $h_{22}:=-\varepsilon_{2}\left\langle\widetilde{\nabla}_{B} B, N\right\rangle$. Since $\nabla$ is determined by $g, h_{22}$ can be computed with the aid of (25) and the Gauss formula (7) giving

$$
h_{22}=\frac{1}{\kappa}\left\{\varepsilon_{3} \frac{\mathscr{F}_{s s}}{\mathscr{F}}-\varepsilon_{2} \tau^{2}+\varepsilon_{1} \varepsilon_{3} \rho\right\} .
$$

Using again the Gauss and Weingarten formulas (7) and (8), it can be shown that $x: U \rightarrow M_{r}^{3}(\rho) \rightarrow \mathbb{E}_{v}^{4}$ satisfies the following PDE system:

$$
\begin{aligned}
x_{s s}= & -\frac{\kappa}{\mathscr{F}} x_{s} \times x_{t}-\varepsilon_{1} \rho x, \\
x_{t s}= & \frac{\mathscr{F}_{s}}{\mathscr{F}} x_{t}-\frac{\tau \mathscr{F}}{\kappa} x_{s s}-\varepsilon_{1} \frac{\tau \mathscr{F}}{\kappa} \rho x, \\
x_{t t}= & -\varepsilon_{1} \varepsilon_{3} \mathscr{F} \mathscr{F}_{s} x_{s}-\varepsilon_{2} \frac{h_{22} \mathscr{F}^{2}}{\kappa} x_{s s} \\
& -\left(\frac{\varepsilon_{1} \varepsilon_{2} h_{22}}{\kappa}+\varepsilon_{3}\right) \mathscr{F}^{2} \rho x+\frac{\mathscr{F}_{t}}{\mathscr{F}} x_{t} .
\end{aligned}
$$

This system can be expressed equivalently in terms of the time variation of the Frenet frame. In fact, from the Gauss formula (10) and (30), we have

$$
\frac{D T}{d t}=\widetilde{\nabla}_{\partial / \partial t} T=-\varepsilon_{2} \mathscr{F} \tau N+\mathscr{F}_{s} B .
$$

So, differentiating $x_{t}=\mathscr{F} B$ and using once more Gauss and Weingarten formulas, we have

$$
x_{t t}=\mathscr{F}_{t} B+\mathscr{F}\left(\widetilde{\nabla}_{\partial / \partial t} B-\varepsilon_{3} \rho \mathscr{F} x\right),
$$

which combined with (32) gives

$$
\frac{D B}{d t}=\widetilde{\nabla}_{\partial / \partial t} B=-\varepsilon_{1} \varepsilon_{3} \mathscr{F}_{s} T-h_{22} \mathscr{F} N .
$$

Finally, from (33), (35), and the cross product relations (18), one has

$$
\frac{D N}{d t}=\widetilde{\nabla}_{\partial / \partial t} N=\varepsilon_{1} \tau \mathscr{F} T+\varepsilon_{2} \varepsilon_{3} h_{22} \mathscr{F} B
$$

which ends the proof.

Now, combining (9) and Gauss and Codazzi equations (10) and (11) with (13), (25), (26), and (27), we obtain after a long computation

$$
\begin{aligned}
\varepsilon_{2} \varepsilon_{3} \frac{\mathscr{F}_{s s}}{\mathscr{F}} & =\varepsilon_{2} \kappa h_{22}+\tau^{2}-\varepsilon_{1} \varepsilon_{2} \varepsilon_{3} \rho, \\
\kappa_{t} & =-2 \mathscr{F}_{s} \tau-\tau_{s} \mathscr{F}, \\
\tau_{t} & =\varepsilon_{1} \varepsilon_{3} \kappa \mathscr{F}_{s}+\varepsilon_{2}\left(h_{22} \mathscr{F}\right)_{s} .
\end{aligned}
$$

Notice that the Gauss equation (37) is equivalent to (29). By substitution of (29) in (39) we get that the Codazzi equations (38) and (39) for $(U, x)$ boil down to

$$
\begin{aligned}
\kappa_{t} & =-2 \mathscr{F}_{s} \tau-\tau_{s} \mathscr{F}, \\
\tau_{t} & =\varepsilon_{1} \varepsilon_{3} \kappa \mathscr{F}_{s}+\varepsilon_{2}\left(\frac{\mathscr{F}}{\kappa}\left(\varepsilon_{3} \frac{\mathscr{F}_{s s}}{\mathscr{F}}-\varepsilon_{2} \tau^{2}+\varepsilon_{1} \varepsilon_{3} \rho\right)\right)_{s} .
\end{aligned}
$$

Observe that if $\mathscr{F}=\kappa,(40)$ and (41) are precisely Da Rios equations for the vortex filament [2]. Moreover, (40) and (41) are the compatibility conditions of the PDE system (30)-(32). Thus, from the fundamental theorem of submanifolds ([8], $\$ 2.7)$, given functions $\kappa(s, t), \tau(s, t)$, and $\mathscr{F}(s, t)$, smoothly defined on a connected domain $U$ and satisfying (40) and (41), there exists a solution of (30)-(32) (and, consequently, of (20)-(21)) determining a smooth isometric immersion $(U, x)$ (unique up to rigid motions, if $U$ is simply connected) of a surface in $M_{r}^{3}(\rho)$ whose metric and the second fundamental form are given, respectively, by $g=\varepsilon_{1} d s^{2}+\varepsilon_{3} \mathscr{F}^{2} d t^{2}$ and $\varepsilon_{2} h=-\kappa d s^{2}+2 \tau \mathscr{F} d s d t+\varepsilon_{2} \mathscr{F}^{2} h_{22} d t^{2}$, where $h_{22}$ is obtained from (29).

Computing the Christoffel symbols from the metric coefficients for such an immersion, $(U, x)$, we see that $\gamma^{t}(s)$ are arc-length parametrized geodesics $\forall t$. Then, a combination of the Gauss formula (7) and (15)-(17) for the Frenet frame along the coordinate curves $\gamma^{t}(s),\left\{x_{s}=T(s, t), N(s, t), B(s, t)\right\}$, shows that the unit Frenet normals $N(s, t)$ are perpendicular to the surface $(U, x)$. Hence, $x_{t}=\lambda(s, t) B(s, t)$, but then the second coefficient of $g(23)$ implies that $\mathscr{F}(s, t)=\lambda(s, t)$ and $(U, x)$ is a solution of (19). Since $(U, x)$ is foliated by geodesics $\gamma^{t}(s)$ having $\kappa^{t}(s):=\kappa(s, t)$ and $\tau^{t}(\mathrm{~s}):=\tau(s, t)$ as curvature and torsion, respectively, the immersion itself, $x$, is geometrically determined by $\kappa^{t}(s):=\kappa(s, t)$ and $\tau^{t}(s):=\tau(s, t)$, because, from the fundamental theorem of curves, for any fixed $t$, there exists a unique curve $\gamma^{t}(s)$ (up to congruences and causal character of the Frenet frame) having $\kappa^{t}(s)$ and $\tau^{t}(s)$ as curvature and torsion. Then, smooth assembling of these curves $\gamma^{t}(s), t \in J$, would give $x$. So, geometrically solving (19) amounts to solving system (40), (41) 
Another consequence of the Gauss-Codazzi equations (40) and (41) is that, besides length, other geometric quantities may also be invariant for closed filaments. More precisely, we have the following.

Proposition 2. Let $(U, x)$ be a binormal evolution surface in $M_{r}^{3}(\rho)$ with velocity $\mathscr{F}$ and assume that $s \in[0,1]$ and all filament curves $\gamma^{t}(s)=x(s, t)$ are $C^{4}$-closed in $[0,1]$. If $\mathscr{F}(\kappa, \tau)=\mathscr{F}(\kappa)$, then $\int_{0}^{1} \tau d s$ and $\int_{0}^{1}(P(\kappa)+\eta \tau) d s$, with $d P / d \kappa=\mathscr{F}(\kappa), \eta \in \mathbb{R}$, are independent of $t$. Furthermore, if $\mathscr{F}(\kappa)$ is constant, then also $\int_{0}^{1} \kappa d s$ is invariant. Finally, if $\mathscr{F}(\kappa, \tau)=n \kappa+m \tau$, then $\int_{o}^{1}\left(m \kappa-\varepsilon_{1} \varepsilon_{3} n \tau\right) d s$ does not depend on $t$.

Proof. Only first part is proved since the others are similar. If $\mathscr{F}(\kappa, \tau)=\mathscr{F}(\kappa)$, then, the invariance $\int_{0}^{1} \tau d s$ is a direct consequence of $\kappa \mathscr{F}_{s}=(\kappa \mathscr{F}-P)_{s}$, because using (41) we have $(d / d t) \int_{0}^{1} \tau d s=\int_{0}^{1} \tau_{t} d s=0$. Now, from $(40)$

$$
\begin{aligned}
& \frac{d}{d t} \int_{0}^{1}(P(\kappa)-\eta \tau) d s=\int_{0}^{1} \kappa_{t} \mathscr{F} d s-\eta \int_{0}^{1} \tau_{t} d s \\
& \quad=\int_{0}^{1}\left(\mathscr{F}^{2} \tau\right)_{s} d s=0 .
\end{aligned}
$$

\section{Evolution with Constant Torsion}

Now we study binormal evolution surfaces, whose filaments have the same constant torsion. Since $\tau=\tau_{o} \in \mathbb{R}, \mathscr{F}(s, t)=$ $\mathscr{F}\left(\kappa(s, t), \tau_{o}\right):=\widetilde{F}(\kappa(s, t))$. Choose $P(u)$ so that $\dot{P}(u):=$ $d P / d u=\widetilde{\mathscr{F}}(u)$. Assume first $\tau=0$.

Proposition 3. Let $S_{\gamma}$ be a binormal evolution surface, whose filaments satisfy $\tau=0$. Then $\gamma$ is extremal for the energy $\Theta(\gamma):=\int_{\gamma}(P(\kappa)+\lambda) d s$ and $S_{\gamma}=\left\{\phi_{t}(\gamma), t \in \mathbb{R}\right\}$, where $\left\{\phi_{t}, t \in \mathbb{R}\right\}$ is a 1-parameter group of isometries of $M_{r}^{3}(\rho)$. Moreover, the fibers of $S_{\gamma}$ have constant curvature and zero torsion (if they are not geodesics) in $M_{r}^{3}(\rho)$. In particular, if $\rho=0, S_{\gamma}$ are either ruled surfaces or rotational surfaces.

Proof. By substituting $\tau=0$ in (40) we have $\kappa(s, t)=\kappa(s)$ and the metric with respect to the chosen coordinate system is $g=$ $\varepsilon_{1} d s^{2}+\varepsilon_{3} \widetilde{\mathscr{F}}^{2}(s) d t^{2}$. This means that $(U, x)$ is a warped product surface [8], and since $\partial g_{i j} / \partial t=0$, we have that $x_{t}(s, t)=$ $\widetilde{\mathscr{F}}(s) B(s, t)$ is a Killing field of $(U, x)$. Now, integrating (41) we get

$$
0=\dot{P}_{s s}+\varepsilon_{1} \varepsilon_{2} \dot{P}\left(\kappa^{2}+\varepsilon_{2} \rho\right)-\varepsilon_{1} \varepsilon_{2} \kappa(P+\lambda),
$$

for some $\lambda \in \mathbb{R}$. Moreover, since $\tau=0$, we have that (43) is the Euler-Lagrange equation for $\Theta(\gamma)([9,10])$ and $\gamma^{t}$ must be an extremal of $\Theta$ in $M_{r}^{2}(\rho), \forall t$. On the other hand, for a given field along $\gamma, W$, the following variation formulas for $v=|\dot{\gamma}|$, $\kappa$, and $\tau$ can be obtained using standard computations and the Frenet equations (see, $[9,10]$ )

$$
\begin{aligned}
W(v)= & \varepsilon_{1} v\left\langle\widetilde{\nabla}_{T} W, T\right\rangle \\
W(\kappa)= & \left\langle\widetilde{\nabla}_{T}^{2} W, N\right\rangle-2 \varepsilon_{1} \kappa\left\langle\widetilde{\nabla}_{T} W, T\right\rangle+\varepsilon_{1} \rho\langle W, N\rangle \\
W(\tau)= & \varepsilon_{2}\left(\frac{1}{\kappa}\left\langle\widetilde{\nabla}_{T}^{2} W+\varepsilon_{1} \rho W, B\right\rangle\right)_{s}-\varepsilon_{1} \tau\left\langle\widetilde{\nabla}_{T} W, T\right\rangle \\
& +\varepsilon_{1} \kappa\left\langle\widetilde{\nabla}_{T} W, B\right\rangle
\end{aligned}
$$

where the subscript $s$ denotes differentiation with respect to the arc-length. Now, combining (43), (44), and the Frenet equations, one can see that

$$
\mathscr{I}(v)=\mathscr{I}(\kappa)=\mathscr{I}(\tau)=0,
$$

along $\gamma$, where $\mathscr{I}=\dot{P} B$. This means that $\mathscr{I}=\dot{P} B$ is a Killing field along $\gamma([9-11])$, but this field is precisely $x_{t}=$ $\widetilde{\mathscr{F}} B$. Now, (44) imply that the Killing vector fields along a curve $\gamma$ form a six-dimensional linear space. Moreover, the Lie algebra of $M_{r}^{3}(\rho)$ is six-dimensional and the restriction of a Killing vector field in $M_{r}^{3}(\rho)$ to any curve $\gamma$ gives a Killing vector field along $\gamma$. Hence, every Killing vector field along a curve, $\gamma$, is the restriction to $\gamma$ of a Killing vector field of $M_{r}^{3}(\rho)[11]$; in other words, $x_{t}$ can be extended to a Killing field on $M_{r}^{3}(\rho)$ (denoted also by $\mathscr{I}$ ). Hence, the associated 1 -parameter group $\left\{\phi_{t}, t \in \mathbb{R}\right\}$ is formed by isometries of $M_{r}^{3}(\rho)$ and $S_{\gamma}=x(U)$ is obtained as $S_{\gamma}=\left\{\phi_{t}(\gamma(s)), t \in \mathbb{R}\right\}$, where $\gamma(s)=x(s, 0)$. Since $x_{s}=T(s, t), x_{t}=\dot{P}(\kappa(s)) B(s, t)$, and $\widetilde{\nabla}_{x_{s}} B(s, t)=-\varepsilon_{2} \tau N(s, t)=0$, we get that $B(s, t)$ does not depend on $s$. Moreover, as fibers are orbits of a Killing field of $M_{r}^{3}(\rho)$, they have constant curvature. Now, for any $s_{o}$ take an arc-length parametrization, $\delta(t)$, of the fiber of $S_{\gamma}$ through $s_{o}$. With the subscript $\delta$ denoting the geometric elements associated with the curve $\delta$, we have $T_{\delta}(t)=B\left(t / \dot{P}\left(\kappa\left(s_{o}\right)\right)\right)$ and, using the last equation of (21), we obtain

$$
\varepsilon_{2}^{\delta} \kappa_{\delta} N_{\delta}=-\varepsilon_{1} \varepsilon_{3} \frac{\dot{P}_{s}}{\dot{P}} T-h_{22} N
$$

if $\delta$ has nonnull acceleration. Thus, differentiating (46) with respect to $t$ and using again (21), we have that $\delta(t)$ must verify

$$
\begin{aligned}
\kappa_{\delta}(s, t) & =\kappa_{\delta}(s), \\
\kappa_{\delta} \tau_{\delta} & =0, \\
\varepsilon_{2}^{\delta} \kappa_{\delta}^{2} & =\varepsilon_{1} \frac{\dot{P}_{s}^{2}}{\dot{P}^{2}}+\varepsilon_{2} h_{22}^{2},
\end{aligned}
$$

from which we see that either $\kappa_{\delta}=0$ and $\delta(t)$ is a geodesic in $M_{r}^{3}(\rho)$ or $\kappa_{\delta} \neq 0$ and $\delta(t)$ is a planar circle.

On the other hand, if $\delta$ has null acceleration and is not a geodesic, then we can consider the following frame along $\gamma$. Define $N_{\delta}(t)$ as the lightlike field on $\delta$ given by $\left(D T_{\delta} / d t\right)(t)=$ $N_{\delta}(t)$ and denote by $B_{\delta}(t)$ the only lightlike vector such that 
$\left\langle N_{\delta}, B_{\delta}\right\rangle=1$ and $\left\langle T_{\delta}, B_{\delta}\right\rangle=0$. In this case, we have the following equations:

$$
\begin{aligned}
& \frac{D T_{\delta}}{d t}(t)=N_{\delta}(t), \\
& \frac{D N_{\delta}}{d t}(t)=\tau_{\delta}(t) N_{\delta}(t), \\
& \frac{D N_{\delta}}{d t}(t)=-T(t)-\tau_{\delta}(t) B_{\delta}(t),
\end{aligned}
$$

for certain function $\tau_{\delta}(t)$ which will be also called the torsion of $\gamma$ (here, the "curvature" is considered to be 1 ). Then, from the second equation of $(21)$, it is clear that $\tau_{\delta}(t)=0$.

Finally, we restrict ourselves to flat ambient spaces $\mathbb{R}^{3}$ or $\mathbb{L}^{3}$. For simplicity, we take first $M_{r}^{3}(\rho)=\mathbb{R}^{3}$. If $P(\kappa)=\nu \kappa, \nu \in$ $\mathbb{R}$, then any planar curve $\gamma$ is critical for $\Theta[12]$ and $S_{\gamma}$ must be a right cylinder shaped on $\gamma$. Assume then that $P(\kappa) \neq \nu \kappa$, $\nu \in \mathbb{R}$. Then the Killing field along $\gamma, \mathscr{I}$, can be written as $\mathscr{I}=\dot{P} B=\lambda_{1}(\gamma \times V)+\lambda_{2} V$, for some $\lambda_{1}, \lambda_{2} \in \mathbb{R}, \lambda_{1} \neq 0$, and a constant vector $V$ in $\mathbb{R}^{3}$. By scalar multiplication of $V$ with the covariant derivative of $\mathscr{I}=\dot{P} B=\lambda_{1}(\gamma \times V)+\lambda_{2} V$ along $\gamma$, we obtain $\lambda_{2}=0$ and, then, $S_{\gamma}$ is a rotational surface in $\mathbb{R}^{3}$ with profile curve $\gamma$. These facts can be extended to the Minkowski space $\mathbb{L}^{3}$ by similar arguments.

Moreover, if $P(\kappa) \neq \nu \kappa, \nu \in \mathbb{R}$, it is not difficult to see that, identifying the plane containing $\gamma$ and $\mathbb{R}^{2}$, choosing a coordinate system containing $V$, and using $\mathscr{I}=\dot{P} B$, it is possible to find a coordinate system in $\mathbb{R}^{2}$ where $\gamma=\left(\gamma_{1}, \gamma_{2}\right)$ and $\gamma_{1}=\eta \dot{P}$, for some constant $\eta \in \mathbb{R}$. This also works in the Minkowski space $\mathbb{L}^{3}$. In particular, take an extremal curve for $\int_{\gamma}(P(\kappa)+\lambda) d s$ either in $\mathbb{R}^{2}$ or in $\mathbb{L}^{2}$ and choose a coordinate system where $\gamma=\left(\gamma_{1}, \gamma_{2}\right)$ and $\gamma_{1}=\eta \dot{P}=\eta \widetilde{\mathscr{F}}$, for some constant $\eta \in \mathbb{R}$. Assuming $\eta \neq 0$, then

$$
\begin{aligned}
& x(s, t)=(\eta \widetilde{\mathscr{F}} \sin (t), \eta \widetilde{\mathscr{F}} \cos (t), a(s)), \\
& \quad \text { if } \varepsilon_{1}^{\delta} \varepsilon_{2}^{\delta}=1, \\
& x(s, t)=(\eta \widetilde{\mathscr{F}} \sinh (t), a(s), \eta \widetilde{\mathscr{F}} \cosh (t)), \\
& \quad \text { if } \varepsilon_{1}^{\delta}=-1, \\
& x(s, t)=(\eta \widetilde{\mathscr{F}} \cosh (t), a(s), \eta \widetilde{\mathscr{F}} \sinh (t)),
\end{aligned}
$$

$$
\text { if } \varepsilon_{2}^{\delta}=-1
$$

where $a(s)$ verifies that $\varepsilon_{2}^{\delta}\left((\eta \widetilde{\mathscr{F}})^{\prime}\right)^{2}+\varepsilon_{3}^{\delta}\left(a^{\prime}(s)\right)^{2}=\varepsilon_{1}$ are rotation surfaces with planar filaments evolving by (19). If the extremal curve lies in the Minkowski plane $\mathbb{L}^{2}$, then choosing the same coordinate system as before, one could also construct a surface with planar filaments evolving by (19) by rotating $\gamma$ around a lightlike axis. In fact, suppose without loss of generality that the lightlike axis is determined by $\partial / \partial x_{3}+\partial / \partial x_{4}$; then, the parametrization of $S_{\gamma}$ is given by

$$
\begin{aligned}
& x(s, t)=\left(\eta \widetilde{F}+(a(s)-\eta \widetilde{F}) \frac{t^{2}}{2},(a(s)-\eta \widetilde{F}) t, a(s)\right. \\
& \left.\quad+(a(s)-\eta \widetilde{F}) \frac{t^{2}}{2}\right),
\end{aligned}
$$

where $a(s)$ verifies $\left((\eta \widetilde{\mathscr{F}})^{\prime}\right)^{2}-\left(a^{\prime}(s)\right)^{2}=\varepsilon_{1}$. In this case, fibers are spacelike curves with null acceleration.

We remark that a periodic solution $\kappa(s)$ of (43) does not determine a closed filament necessarily, and closure conditions have to be derived. For instance, if $\kappa(s)$ is a periodic solution of (19) with period $\rho$ and $\gamma(s)$ is the corresponding curve in $\mathbb{R}^{2}$, then integrating (43) we have $d=\dot{P}_{s}^{2}+(\kappa \dot{P}-P-\lambda)^{2}$, for some $d \in \mathbb{R}$. Choosing a coordinate system in $\mathbb{R}^{2}$ where $\gamma=\left(\gamma_{1}, \gamma_{2}\right)$ and $\gamma_{1}=(1 / \sqrt{d}) \dot{P}$, we see that $\gamma_{1}$ is also periodic. Using the fact that $\gamma$ is arc-lengthparametrized, we have $\gamma_{2}^{\prime}(s)=(1 / \sqrt{d})(\kappa \dot{P}-P-\lambda)$. Hence, $\gamma$ closes up, if and only if $\int_{o}^{\rho}(\kappa \dot{P}-P-\lambda)=0$.

Take now $\tau_{o} \neq 0$. If $\kappa(s, t)$ is also constant, filaments are Frenet helices. The unit binormal $B$ is a Killing vector field on Frenet helices; hence, their evolution under the $B$-flow satisfies $x_{t}=B$, so we assume $\kappa(s, t)$ is not constant. Then (40) suggests studying travelling wave solutions of the form $\kappa(s-$ $\left.\varepsilon_{1} \varepsilon_{3} \mu t\right), \mu \in \mathbb{R}$, which implies $\widetilde{\mathscr{F}}(\kappa(s, t))=\left(\varepsilon_{1} \varepsilon_{3} \mu / 2 \tau_{0}\right) \kappa+\lambda$, for some $\lambda \in \mathbb{R}$. Call $\iota=s-\varepsilon_{1} \varepsilon_{3} \mu t$. Then, by substitution in (41) we obtain

$$
\begin{aligned}
0= & \frac{\varepsilon_{1} \varepsilon_{2} \mu}{2 \tau_{o}} \kappa_{u}+\frac{\mu}{4 \tau_{o}} \kappa^{3}+\frac{(-1)^{r} \mu}{2 \tau_{o}} \kappa\left(\varepsilon_{1} \varepsilon_{3} \rho-\varepsilon_{2} \tau_{o}^{2}\right) \\
& +\lambda\left((-1)^{r} \rho-\tau_{o}^{2}\right) .
\end{aligned}
$$

Proposition 4. Assume that $\gamma(\iota)$ is a curve in $M_{r}^{3}(\rho)$ with nonconstant curvature and constant torsion $\tau=\tau_{o} \neq 0$ which is an extremal of the energy $\widetilde{\Theta}(\gamma)=\int_{\gamma}\left(\left(\varepsilon_{1} \varepsilon_{3} \mu / 4 \tau_{o}\right) \kappa^{2}+\lambda \kappa+\mu \tau+\right.$ $\eta)$, where $\mu \neq 0$ and $\lambda, \eta \in \mathbb{R}$. Then, there exists a 1-parameter group of isometries of $M_{r}^{3}(\rho),\left\{\phi_{t}, t \in \mathbb{R}\right\}$, such that a suitable parametrization of the surface $S_{\gamma}:=\phi_{t}(\gamma(l))$ is a solution of (19) with $\widetilde{\mathscr{F}}(\kappa(s, t))=\left(\varepsilon_{1} \varepsilon_{3} \mu / 2 \tau_{0}\right) \kappa+\lambda$.

Proof. It is easy to verify from (51) that $\kappa(\iota)$ and $\tau=\tau_{o}$ satisfy the Euler-Lagrange equations for the above energy $\widetilde{\Theta}$ $([9,10])$ for a suitable $\eta \in \mathbb{R}$. Consider $\kappa(\iota)$ a solution of (51) (observe that (51) can be explicitly solved with the aid of Jacobi elliptic functions); then $\kappa(\iota)$ and $\tau=\tau_{o}$ determine a curve $\gamma(\iota)$ in $M_{r}^{3}(\rho)$ which is an extremal for $\widetilde{\Theta}$. Now, the vector field $\mathscr{F}(\iota):=\varepsilon_{1} \varepsilon_{3} \mu T(\iota)+\widetilde{\mathscr{F}}(\iota) B(\iota)$ is a Killing field along $\gamma(\iota)([9,10])$. As in previous proposition, $\mathscr{I}$ can be extended to a Killing field on $M_{r}^{3}(\rho)$ with 1-parameter group of isometries $\left\{\phi_{t}, t \in \mathbb{R}\right\}$. Consider the surface $S_{\gamma}:=y(l, t)=$ $\phi_{t}(\gamma(\iota)), t \in \mathbb{R}$. Then, the reparametrization $x(s, t):=y(s-$ $\left.\varepsilon_{1} \varepsilon_{3} \mu t, t\right)$ satisfies $x_{t}=\left(\left(\varepsilon_{1} \varepsilon_{3} \mu / 2 \tau_{0}\right) \kappa+\lambda\right) B$, all filaments having constant torsion $\tau=\tau_{o}$. 
If $\mu=0$, then $\kappa(s, t)=\kappa(s)$ and $\widetilde{\mathscr{F}}(\kappa(s))=\lambda$ and $S_{\gamma}$ is flat since, combining (10) and (25), we obtain that the Gaussian curvature of $S_{\gamma}$ is given by

$$
\begin{aligned}
K & :=\frac{R(\partial / \partial s, \partial / \partial t, \partial / \partial t, \partial / \partial s)}{g(\partial / \partial s, \partial / \partial s) g(\partial / \partial t, \partial / \partial t)-g(\partial / \partial s, \partial / \partial t)^{2}} \\
& =-\varepsilon_{1} \frac{\mathscr{F}_{s s}}{\mathscr{F}}=0 .
\end{aligned}
$$

Moreover, (51) implies that $\rho=(-1)^{r} \tau_{o}^{2}$. Thus $M_{r}^{3}(\rho)$ has to be either $\mathbb{S}^{3}(\rho)$ or $\mathbb{M}_{1}^{3}(\rho)$. Flat surfaces in $\mathbb{S}^{3}(\rho)$ can be locally described as the product, with respect to the Lie group structure of $\mathbb{S}^{3}(\rho)$, of two curves with torsions $\rho$ and $-\rho$, respectively, [13]. In order to construct explicit parametrizations solving (19) in this case, we take the complex plane, $\mathbb{C}$, and consider the maps $\pi_{\varepsilon}: \mathbb{C}^{2} \rightarrow \mathbb{C}^{2}$ defined by $\pi_{\varepsilon}\left(z_{1}, z_{2}\right)=$ $(1 / 2 c)\left(\bar{z}_{1} z_{1}-\varepsilon \bar{z}_{2} z_{2}, 2 z_{2} \bar{z}_{1}\right)$, where $z_{i} \in \mathbb{C}, i \in\{1,2\}, \bar{z}_{i}$ denotes complex conjugate, $\varepsilon= \pm 1$, and $c \in \mathbb{R}$. Endow $\mathbb{C}^{2}$ with the semi-Riemannian metric $\langle z, w\rangle=\operatorname{Real}\left(z_{1} \bar{w}_{1}+\right.$ $\left.\varepsilon z_{2} \bar{w}_{2}\right)$. Then, the restriction of $\pi_{\varepsilon}$ to the hyperquadrics $\langle z, z\rangle=\varepsilon c^{2}, \varepsilon= \pm 1$, gives two maps which are known as the standard Hopf mappings

$$
\begin{aligned}
& \pi_{+}: \mathbb{S}^{3}\left(\frac{1}{c^{2}}\right) \longrightarrow \mathbb{S}^{2}\left(\frac{4}{c^{2}}\right), \\
& \pi_{-}: \mathbb{W}_{1}^{3}\left(\frac{-1}{c^{2}}\right) \longrightarrow \mathbb{M}^{2}\left(\frac{-4}{c^{2}}\right) .
\end{aligned}
$$

Let $\gamma$ be a curve in either $\mathbb{S}^{2}\left(4 / c^{2}\right)$ or $\mathbb{H}^{2}\left(-4 / c^{2}\right)$. Then, the complete lift $\mathbf{T}_{\gamma}^{+}=\pi_{+}^{-1}(\gamma)$ (resp., $\mathbf{T}_{\gamma}^{-}=\pi_{-}^{-1}(\gamma)$ ) is a Riemannian (resp., Lorentzian) flat (zero Gaussian curvature) surface in $\mathbb{S}^{3}\left(1 / c^{2}\right)$ (resp., in $\mathbb{M}_{1}^{3}\left(-1 / c^{2}\right)$ ) which is called the Hopf cylinder on $\gamma$. The covering maps $\Psi^{+}: \mathbb{R}^{2} \rightarrow \mathbf{T}_{\gamma}^{+}$and $\Psi^{-}: \mathbb{L}^{2} \rightarrow \mathbf{T}_{\gamma}^{-}$

$$
\Psi^{ \pm}(t, s)=e^{i t} \bar{\gamma}(s),
$$

where $\bar{\gamma}(s)$ denotes a horizontal lift of $\gamma$, can be used to parametrize $\mathbf{T}_{\gamma}^{ \pm}$. Assuming without loss of generality $c=1$, that is, $\rho= \pm 1$, critical curves of $\int_{\gamma} \kappa$ in $\mathbb{S}^{3}(1)$ or $\mathbb{U}_{1}^{3}(-1)$ are characterized by having torsion $\tau^{2}=1[5,12]$, and, therefore, they must be horizontal lifts via Hopf maps. Hence, we have the following.

Proposition 5. Horizontal lifts via the Hopf map $\pi_{ \pm}$of arbitrary curves $\gamma$ of $\mathbb{S}^{2}(4)$ or $\mathbb{H}^{2}(-4)$ parametrized by (54) evolve under $x_{t}=B$ by rigid motions and the corresponding binormal evolution surface is a Hopf cylinder of $\mathbb{S}^{3}(1)$ or $\mathbb{M}_{1}^{3}(-1)$ shaped on $\gamma, \mathbf{T}_{\gamma}^{ \pm}$.

Thus, explicit parametrizations of $\mathbf{T}_{\gamma}^{ \pm}$are obtained as follows. Take an arbitrary curve $\gamma(s)=\left(A_{1}(s), 0, A_{2}(s), A_{3}(s)\right)$ in $\mathbb{S}^{2}(4)$ or $\mathbb{M}^{2}(-4)$; then horizontal lifts of $\gamma$ via $\pi_{+}$or $\pi_{-}$are given by

$$
\begin{gathered}
\bar{\gamma}(s)=(M(s) \cos \alpha(s), M(s) \sin \alpha(s), D(s) \cos \alpha(s) \\
-P(s) \sin \alpha(s), D(s) \sin \alpha(s)+P(s) \cos \alpha(s)),
\end{gathered}
$$

where

$$
\begin{aligned}
M(s) & =\frac{\sqrt{2 A_{1}(s)+1}}{\sqrt{2}}, \\
D(s) & =\frac{\sqrt{2} A_{2}(s)}{\sqrt{2 A_{1}(s)+1}}, \\
P(s) & =\frac{\sqrt{2} A_{3}(s)}{\sqrt{2 A_{1}(s)+1}}, \\
\alpha(s) & = \pm 2 \int \frac{A_{3}(s) A_{2}^{\prime}(s)-A_{2}(s) A_{3}^{\prime}(s)}{2 A_{1}(s)+1} .
\end{aligned}
$$

Hence, one uses (54) and (55) to obtain a solution of (19). Notice that if the curve $\gamma$ is embedded in either $\mathbb{S}^{2}(4)$ or $\mathbb{H}^{2}(-4)$, then so is $\mathbf{T}_{\gamma}^{ \pm}$in $\mathbb{S}^{3}(1)$ or $\mathbb{U}_{1}^{3}(-1)$ and we have binormal Hopf cylinders with no self-intersections. Moreover, if $\gamma$ is a closed curve then $\mathbf{T}_{\gamma}^{ \pm}$is a closed surface (a flat Hopf Tori) but the evolving filament $\bar{\gamma}(s)$ is not closed because of the nontrivial holonomy. However, if, in addition, the area enclosed by $\gamma(s)$ in either $\mathbb{S}^{2}(4)$ or $\mathbb{H}^{2}(-4)$ is a rational multiple of $\pi$, then there are $m \in \mathbb{Z}$ such that the horizontal lift of an $m$-cover of $\gamma(s)$ is a closed filament [12].

\section{Conflicts of Interest}

The authors declare that they have no conflicts of interest.

\section{Acknowledgments}

This research was supported by MINECO-FEDER Grant MTM2014-54804-P, Gobierno Vasco Grant IT1094-16, and UPV/EHU GIU13/08, Spain. Álvaro Pámpano has been supported by Programa Predoctoral de Formación de Personal Investigador No Doctor, Gobierno Vasco, 2015.

\section{References}

[1] Q. Ding, X. Liu, and W. Wang, "The vortex filament in the Minkowski 3-space and generalized bi-Schrödinger maps," Journal of Physics. A. Mathematical and Theoretical, vol. 45, no. 45, Article ID 455201, 455201, 14 pages, 2012.

[2] L. S. da Rios, "Sul moto d'un liquido indefinito con un filetto vorticoso di forma qualunque," Rendiconti del Circolo Matematico di Palermo, vol. 22, no. 1, pp. 117-135, 1906.

[3] W. K. Schief and C. Rogers, "Binormal motion of curves of constant curvature and torsion. Generation of soliton surfaces," The Royal Society of London. Proceedings. Series A. Mathematical, Physical and Engineering Sciences, vol. 455, no. 1988, pp. 31633188, 1999.

[4] S. Kida, "A vortex filament moving without change of form," Journal of Fluid Mechanics, vol. 112, pp. 397-409, 1981.

[5] M. Barros, A. Ferrández, P. Lucas, and M. A. Meroño, "Solutions of the Betchov-Da Rios soliton equations: a Lorentzian approach," Journal of Geometry and Physics, vol. 31, pp. 217-228, 1999.

[6] N. Koiso, "Vortex filament equation in a Riemannian manifold," Tohoku Mathematical Journal, vol. 55, no. 2, pp. 311-320, 2003. 
[7] O. J. Garay and A. Pámpano, "Binormal evolution of curves with prescribed velocity," WSEAS transactions on fluid mechanics 11, pp. 112-120, 2016.

[8] B.-Y. Chen, Pseudo-Riemannian geometry, $\delta$-invariants and applications, World Scientific, 2011.

[9] J. Arroyo, Presión Calibrada Total. Estudio Variacional Y Aplicaciones Al Problema De Chen-Willmore, University of the Basque Country, Spain, 2001.

[10] A. Ferrández, J. Guerrero, M. A. Javaloyes, and P. Lucas, "Particles with curvature and torsion in three-dimensional pseudo-Riemannian space forms," Journal of Geometry and Physics, vol. 56, no. 9, pp. 1666-1687, 2006.

[11] J. Langer and D. A. Singer, "The total squared curvature of closed curves," Journal of Differential Geometry, vol. 20, no. 1, pp. 1-22, 1984.

[12] J. Arroyo, M. Barros, and O. J. Garay, "Some examples of critical points for the total mean curvature functional," Proceedings of the Edinburgh Mathematical Society. Series II, vol. 43, no. 3, pp. 587-603, 2000.

[13] J. A. Gálvez, "Surfaces of constant curvature in 3-dimensional space forms," Math. Contemporânea, vol. 37, pp. 1-42, 2009. 


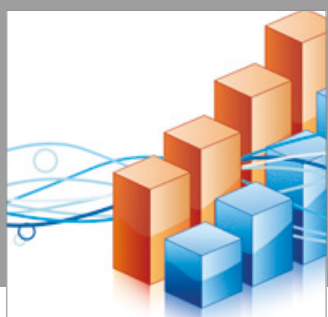

Advances in

Operations Research

vatersals

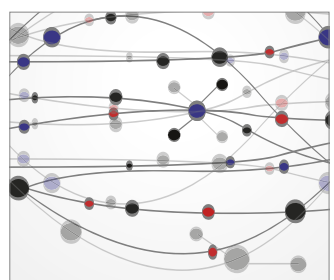

\section{The Scientific} World Journal
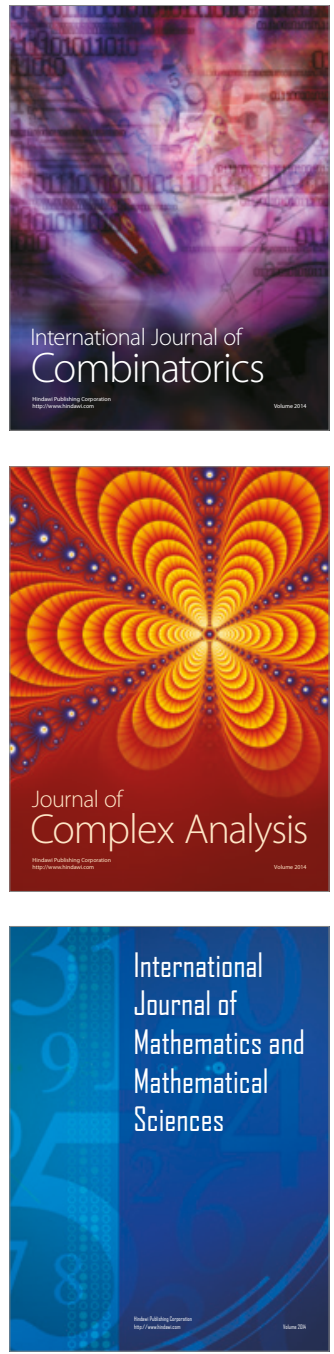
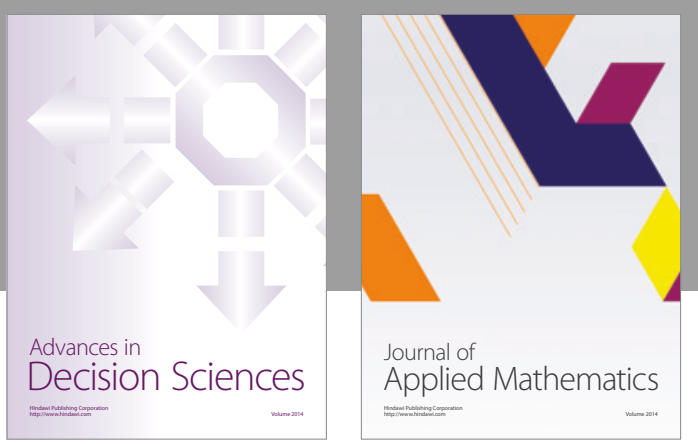

Algebra

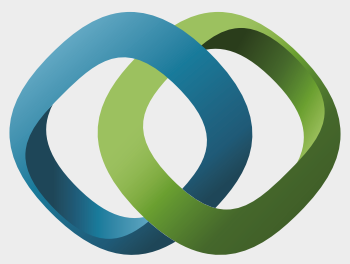

\section{Hindawi}

Submit your manuscripts at

https://www.hindawi.com
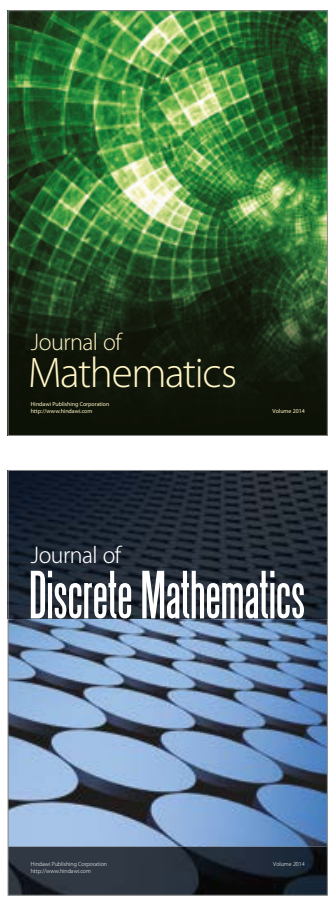

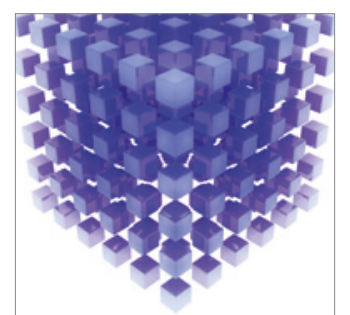

Mathematical Problems in Engineering
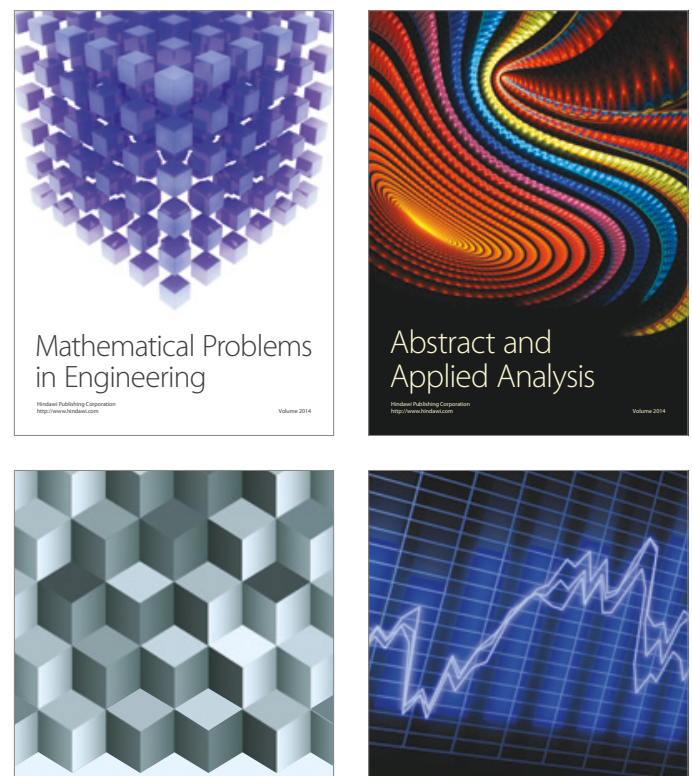

Journal of

Function Spaces

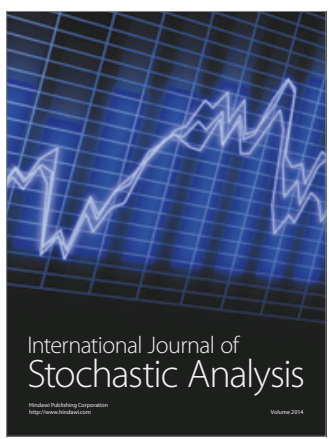

Probability and Statistics
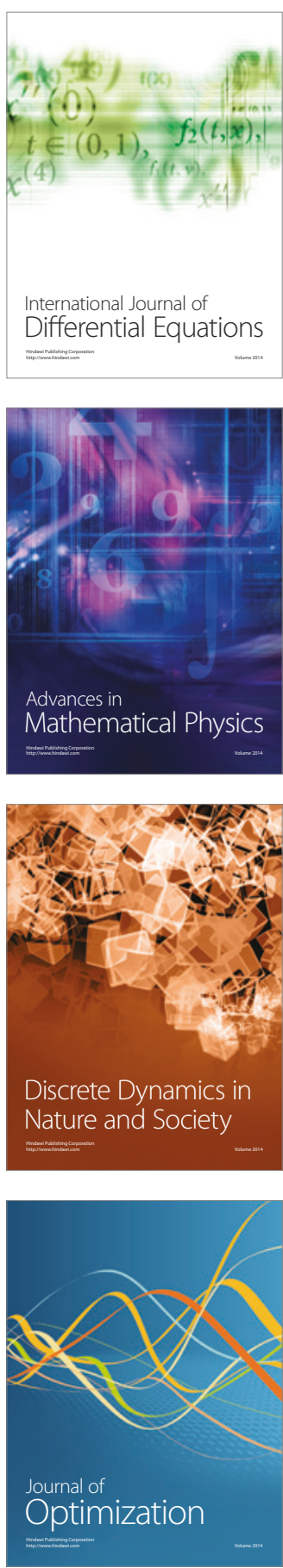